\title{
Antibiotic susceptibility of Helicobacter pylori in Germany: stable primary resistance from 1995 to 2000
}

\author{
KATHLEN WOLLE, ANDREAS LEODOLTER*, PETER MALFERTHEINER* and WOLFGANG KÖNIG \\ Institute of Medical Microbiology and *Department of Gastroenterology, Hepatology and Infectious Diseases, \\ Otto-von-Guericke-University Magdeburg, Germany
}

\begin{abstract}
The issue of antibiotic resistance in Helicobacter pylori is of particular concern and has become an important factor leading to eradication failure. This paper reports the prevalence of primary resistance to clarithromycin, amoxicillin, metronidazole and tetracycline among $H$. pylori isolates in the north-eastern part of Germany. A total of 1644 clinical $H$. pylori isolates was investigated over a period of 6 years from 1995 to 2000. The MICs were determined by the Etest. The overall rate of primary resistance was $\mathbf{2 6 . 2} \%$ for metronidazole and $\mathbf{2 . 2 \%}$ for clarithromycin. No significant changes in the resistance rates during the period of investigation were observed. No isolate was resistant to amoxicillin or tetracycline. PCR-RFLP analysis for the detection of point mutations associated with clarithromycin resistance was performed with $36 \mathrm{H}$. pylori isolates. The $\mathrm{A} \rightarrow \mathrm{G}$ transition mutation at position 2143 was detected in $19 \mathrm{H}$. pylori isolates $(52.8 \%)$, whereas the mutation at position 2142 was found in 13 isolates $(36.1 \%)$.
\end{abstract}

\section{Introduction}

Therapy of Helicobacter pylori infection has become the undisputed first option for all patients with peptic ulcers and is advised for several other associated clinical conditions [1]. Eradication treatment usually consists of a proton pump inhibitor in combination with several antimicrobial agents. Those commonly used for the treatment of $H$. pylori infections include clarithromycin, amoxicillin, metronidazole and tetracycline. An increase in resistance rates to these antimicrobial agents is to be expected because of the increasing numbers of patients treated, imperfect patient compliance and increasing consumption of antibiotics in recent years $[2,3]$. The principal reason for failure to eradicate infection is believed to be the combination of resistance of $H$. pylori with poor patient compliance. Reported prevalences of resistance to clarithromycin, amoxicillin and metronidazole vary widely among geographic areas [2, 4]. Knowledge of antibiotic resistance rates is important because treatment of $H$. pylori infection is started with a standard

Received 13 Nov. 2001; revised version received 29 Jan 2002; accepted 14 March 2002.

Corresponding author: Professor Dr W. König (e-mail: wolfgang.koenig@medizin.unimagdeburg.de). therapeutic regimen. Primary resistance decreases the success of $H$. pylori eradication, hence the local prevalence of resistance should be monitored [5].

For this reason the aims of the present study were: (i) to evaluate the prevalence of primary resistance to clarithromycin, amoxicillin, metronidazole and tetracycline among $H$. pylori isolates in the north-eastern part of Germany and to monitor changes over a 6-year period, (ii) to determine the distribution of MICs of these antimicrobial agents against $H$. pylori strains and (iii) to analyse the point mutations associated with resistance to clarithromycin.

\section{Materials and methods}

\section{H. pylori isolates and growth conditions}

A total of 1644 clinical isolates of $H$. pylori (909 from female and 735 from male patients; average age 43.2 years, range 19-78 years) obtained from 1995 to 2000 was included in this study. H. pylori was cultured from gastric biopsies (either from the antrum or the corpus) of patients who underwent a diagnostic gastroduodenoscopy in the Department of Gastroenterology, Hepatology and Infectious Diseases. Isolates were from patients with chronic gastritis (1403 cases), 
peptic ulcer (221 patients) and gastric cancer (20 cases). None of the patients had been treated with metronidazole or clarithromycin for the eradication of H. pylori infection.

H. pylori was cultured from gastric biopsy specimens on selective medium (Pylori Agar; bioMérieux, France) under micro-aerobic conditions at $37^{\circ} \mathrm{C}$. Plates were examined after 2 days and up to 10 days of incubation. H. pylori was identified by typical morphology on gram-stained smears and positive urease, oxidase and catalase tests. H. pylori ATCC 49503 was used as a positive control.

\section{Determination of MIC}

MICs were determined by the Etest (AB Biodisk, Sweden) on blood agar plates [6]. Suspensions of $H$. pylori were adjusted to a McFarland standard no. 2 as the inoculum. After an incubation period of 3 days under micro-aerobic conditions, the MICs of clarithromycin, amoxicillin, metronidazole and tetracycline were determined. Strains with an MIC of $>8 \mathrm{mg} / \mathrm{L}$ were considered resistant to metronidazole. For clarithromycin, resistance was defined as an MIC of $>1 \mathrm{mg} / \mathrm{L}$ and for amoxicillin and tetracycline as an MIC of $>2 \mathrm{mg} / \mathrm{L}$.

\section{Detection of point mutations associated with clarithromycin resistance}

The point mutations of $H$. pylori were detected by restriction fragment length polymorphism (RFLP) analysis in one PCR-amplified gene segment. Bacterial DNA was extracted from $36 \mathrm{H}$. pylori isolates with phenotypic clarithromycin resistance according to standard procedures. One fragment of the peptidyltransferase region of the $23 \mathrm{~S}$ rRNA of $H$. pylori was amplified by PCR. A primer extending from positions 1820 to 1839 (5'-CCACAGCGATGTGGTCTCAG-3') and a reverse primer from positions 2244 to $2225\left(5^{\prime}\right.$ CTCCATAAGAGCCAAAGCCC-3') were used to amplify a 425-bp DNA fragment [7]. PCR amplification was performed in a final volume of $50 \mu \mathrm{l}$, with $1 \mu \mathrm{g}$ of extracted DNA and $20 \mathrm{pmol}$ of each primer. After $40 \mathrm{PCR}$ cycles with denaturation at $94^{\circ} \mathrm{C}$ for $1 \mathrm{~min}$, annealing at $55^{\circ} \mathrm{C}$ for $1 \mathrm{~min}$, extension at $72^{\circ} \mathrm{C}$ for $1 \mathrm{~min}$ and a final elongation step at $72^{\circ} \mathrm{C}$ for $7 \mathrm{~min}$, PCR products were analysed on agarose $2 \%$ gels.

The amplified DNA was precipitated with ethanol and digested with restriction endonuclease $B s a \mathrm{I}$ or $B b s \mathrm{I}$ (New England Biolabs, Beverly, MA, USA). The products were incubated for $24 \mathrm{~h}$ at $50^{\circ} \mathrm{C}$ for $B s a \mathrm{I}$ and at $37^{\circ} \mathrm{C}$ for $B b s \mathrm{I}$ to detect the restriction site occurring with mutations $\mathrm{A} \rightarrow \mathrm{G}$ at position 2143 and position 2142 , respectively. The restriction fragments were electrophoresed in an agarose $3 \%$ gel.

\section{Statistical analysis}

Differences in the proportions of patients with susceptible and resistant $H$. pylori strains in each year were analysed by Fisher's exact test with a 95\% confidence interval. The frequencies of resistant $H$. pylori isolates from female and male patients and from patients with different clinical manifestations were also compared.

\section{Results}

The overall rates of primary resistance to metronidazole and clarithromycin in $1644 \mathrm{H}$. pylori individual patient isolates were $26.2 \%$ (95\% CI $24.1-28.3 \%)$ and $2.2 \%$ (95\% CI $1.5-2.9 \%)$, respectively (Table 1$) ; 21$ $(58.3 \%)$ of the 36 isolates resistant to clarithromycin were also resistant to metronidazole. No resistance to amoxicillin or tetracycline was observed. There were no significant changes in the prevalence of primary metronidazole and clarithromycin resistance during the period of investigation (Fig. 1). The MIC50 and MIC90 values and the MIC ranges for each antibiotic are summarised in Table 2.

Metronidazole resistance was more frequent in $H$. pylori isolates from female than male patients $(32.2 \%$ versus $20.8 \%$ ), but this did not reach statistical significance $(\mathrm{p}>0.05)$. Primary clarithromycin resistance was similar in $H$. pylori isolates from female and male patients $(2.4 \%$ versus $2.0 \%)$.

No significant differences were observed in the prevalence of metronidazole and clarithromycin resistance between $H$. pylori isolates from patients with chronic gastritis and peptic ulcer.

PCR-RFLP analysis for the study of point mutations associated with clarithromycin resistance was performed for $36 \mathrm{H}$. pylori isolates. In $19 \mathrm{H}$. pylori isolates $(52.8 \%)$ the $\mathrm{A} \rightarrow \mathrm{G}$ transition mutation at position 2143 was detected, whereas the mutation at position 2142 was found in $13 \mathrm{H}$. pylori isolates (36.1\%). In four cases it was not possible to detect the mutations by restriction with $B s a \mathrm{I}$ and $B b s \mathrm{I}$. These strains possibly carry a transversion mutation of $\mathrm{A} \rightarrow \mathrm{C}$, which the restriction analysis would not detect.

Table 1. Overall antibiotic resistance of $H$. pylori isolates from 1644 patients

\begin{tabular}{lcc}
\hline Agents & $\begin{array}{c}\text { Number }(\%) \text { of } \\
\text { resistant isolates }\end{array}$ & $95 \%$ CI \\
\hline Amoxicillin & $0(0)$ & $0.0-2.0$ \\
Clarithromycin & $36(2.2)$ & $1.5-2.9$ \\
Metronidazole & $431(26.2)$ & $24.1-28.3$ \\
Tetracycline & $0(0)$ & $0.0-2.0$ \\
\hline
\end{tabular}


a

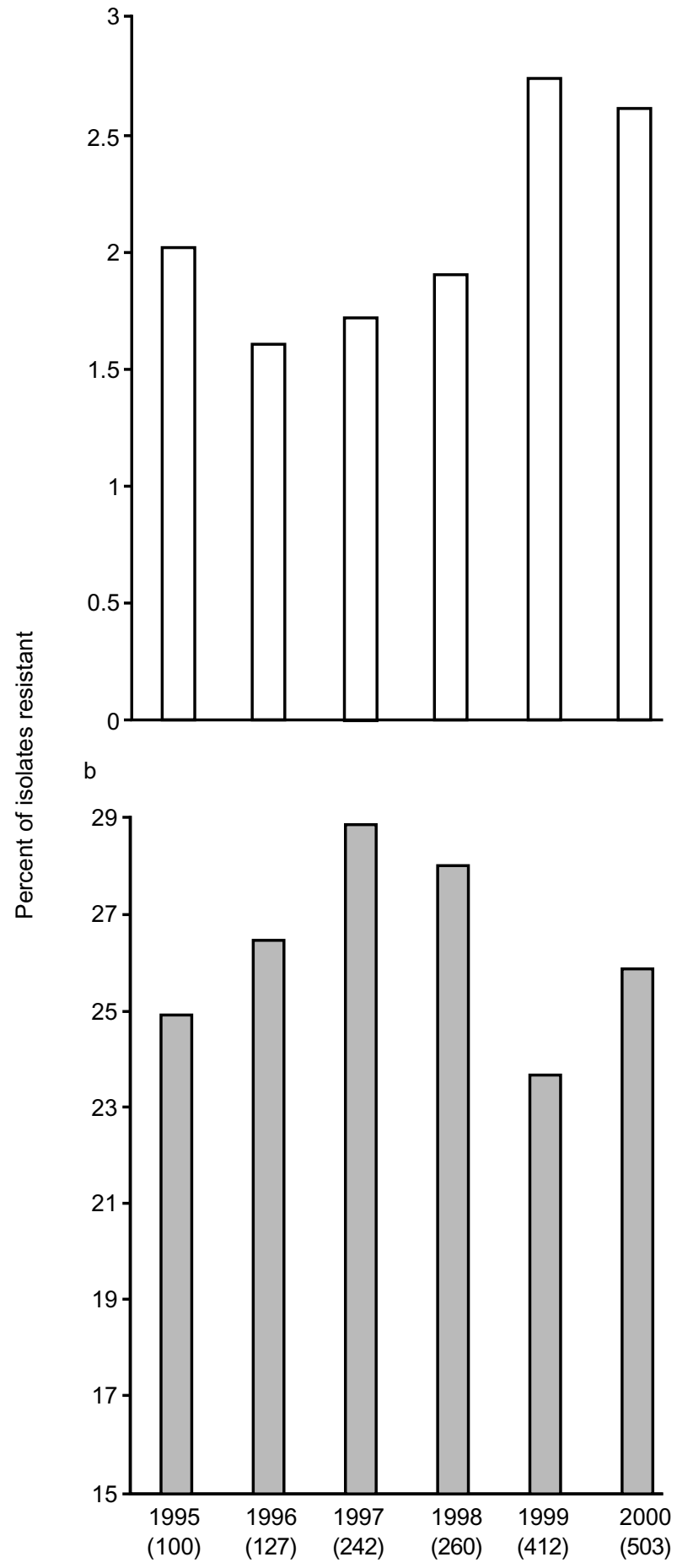

Fig. 1. Percentage of isolates resistant to (a) clarithromycin and (b) metronidazole during the investigating period (19952000). Numbers in parenthesis are the number of isolates examined each year.

Table 2. MICs $(\mathrm{mg} / \mathrm{L})$ of amoxicillin, clarithromycin, metronidazole and tetracycline for 1644 clinical isolates tested

\begin{tabular}{lccc}
\hline Agent & MIC50 & MIC90 & MIC range \\
\hline Amoxicillin & $<0.016$ & 0.023 & $<0.016-0.094$ \\
Clarithromycin & $<0.016$ & 0.064 & $<0.016->256$ \\
Metronidazole & 0.5 & 128 & $0.064->256$ \\
Tetracycline & 0.064 & 0.38 & $<0.016-0.5$ \\
\hline
\end{tabular}

\section{Discussion}

Resistance to antimicrobial agents in $H$. pylori is of particular concern because it is a major determinant in the failure of eradication regimens [8]. Antimicrobial drug resistance has been reported for nitro-imidazoles, macrolides, fluoroquinolones, amoxicillin, rifabutin and tetracyclines. Therefore, susceptibility testing of $H$. pylori is increasingly important. Two types of resistance must be considered: 'primary' resistance supposedly linked to previous exposure of the H. pylori strain to the antibiotic during treatment for another infection unrelated to $H$. pylori, and 'secondary' resistance resulting from treatment aimed at eradicating H. pylori. Resistance to metronidazole is the most common, ranging between $16 \%$ and $42 \%$ in Europe, while the overall prevalence of resistance to macrolides is lower, ranging between $1 \%$ and $12 \%$ in most countries $[2,4,9,10]$.

Nitro-imidazoles were among the first drugs identified as possessing a powerful anti-H. pylori effect. Metronidazole resistance occurs mainly by mutations in the $r d x A$ gene of $H$. pylori, which encodes an enzyme that reduces metronidazole to active metabolites. Mutations in the frxA gene, encoding an $\mathrm{NAD}(\mathrm{P}) \mathrm{H}$ flavin reductase with high homology with the $r d x A$ product can also affect metronidazole susceptibility [11].

In the current investigation the overall rate of primary resistance to metronidazole was $26.5 \%$, which remained stable over the 6 years, and this confirms earlier results reported in 1998 [12]. Results from a European multicentre trial showed the level of overall metronidazole resistance to be $27 \%$, with great variation observed among countries, from 16\% in France to $42 \%$ in Norway [4]. In other studies, the prevalence of metronidazole resistance was reported to be $21 \%$ in the Netherlands, $20 \%$ in Spain and $29 \%$ in Bulgaria $[3,13,14]$. In the USA, metronidazole resistance was $37.4 \%$ in a large study conducted in a metropolitan hospital [15]. An increase in resistance to metronidazole from $30 \%$ to $48 \%$ over 5 years has been reported in Belgium [16], whereas no increase in metronidazole resistance rates occurred in this area of north-Eastern Germany over the last 6 years. The metronidazole MICs showed a normal distribution with an MIC50 of $0.5 \mathrm{mg} / \mathrm{L}$ and an MIC90 of $128 \mathrm{mg} / \mathrm{L}$.

As reported by others, the results of the present study confirm that women are more likely to harbour resistant $H$. pylori strains than men $(32.2 \%$ versus $20.8 \%$, respectively) [2]. This supports the suggestion that resistance of $H$. pylori to metronidazole may partly be due to its frequent use in the treatment of gynaecological infections.

Several macrolides, alone or in combination with other antibiotics and a proton pump inhibitor, have been used in $H$. pylori eradication regimens, but among them 
clarithromycin has been shown to be superior. This compound achieves high concentrations in the gastric mucosa and becomes highly concentrated intracellularly. Analysis of clarithromycin-resistant $H$. pylori strains revealed that point mutation in the peptidyl transferase domain of the 23S rRNA is the mechanism of resistance, and adenine to guanine transition mutations at position 2142 or 2143 were identified [17].

The rate of resistance to clarithromycin is consistently much lower than that to metronidazole. However, based on recent studies the prevalence of clarithromycin resistance varies considerably and ranges from $3.5 \%$ in Spain, to $5.7 \%$ in Italy and up to $7.7 \%$ in Korea $[12,18,19]$. The overall level of primary resistance $(2.2 \%)$ observed in the study population is comparable to that observed in the Netherlands $(1.7 \%)$ [3]. In the present study, the percentage of resistance was $2.6 \%$ in 2000 , which is only marginally higher than the $1.6 \%$ found in 1996. The MIC distribution for clarithromycin in H. pylori was normal with an MIC90 of 0.064 $\mathrm{mg} / \mathrm{L}$. The situation is dramatically different in other countries such as Bulgaria, where clarithromycin resistance increased to $12.5 \%$ over 4 years [9].

Thirty-six clarithromycin-resistant $H$. pylori isolates were analysed by PCR-RFLP to detect point mutations. By restriction with endonuclease $B s a \mathrm{I}$ and $B b s \mathrm{I}$ it was possible to discriminate between the transition mutation $A \rightarrow G$ at positions 2142 and 2143. The mutation at position 2143 was detected in $52.8 \%$ of isolates and the mutation at position 2142 was found in $36.1 \%$. In four cases it was not possible to detect mutations and these strains possibly had an $\mathrm{A} \rightarrow \mathrm{C}$ transversion mutation, without a restriction site. Conventional methods to determine antibiotic susceptibility of $H$. pylori are based on agar dilution testing or the Etest, which are time-consuming and require specific laboratory expertise. Therefore, DNA-based diagnostic methods may offer a rapid and alternative approach for macrolide susceptibility testing and could be useful when exploring the epidemiology of macrolide resistance in $H$. pylori [20].

In the present study all strains were susceptible to amoxicillin and tetracycline. These results confirm that amoxicillin-tolerant $H$. pylori strains identified by Dore and colleagues are still exceptional [21].

In conclusion, primary resistance rates for clarithromycin and metronidazole were stable over the last 6 years in the study population. The prevalence of metronidazole resistance is similar to the median rates in Europe, and the rate of clarithromycin resistance is still low compared with other areas. No resistance to amoxicillin or tetracycline was observed during the period of investigation. Therefore, clarithromycin-based triple therapy can still be recommended as first-line therapy for $H$. pylori eradication in the north-eastern part of Germany. Susceptibility testing of H. pylori isolates in different geographical areas is advisable because it is an aid to selection of optimal therapy regimens.

\section{References}

1. The European Helicobacter pylori Study Group (EHPSG). Current European concepts in the management of Helicobacter pylori infection. The Maastricht 2-2000 Consensus Report. Aliment Pharmacol Ther 2002; 16: 167-180.

2. Canton R, De Argila MC, De Rafael L, Baquero F. Antimicrobial resistance in Helicobacter pylori. Rev Med Microbiol 2001; 12: 47-61.

3. Debets-Ossenkopp YJ, Herscheid AJ, Pot RGJ, Kuipers EJ, Kusters JG, Vandenboucke-Grauls CMJE. Prevalence of Helicobacter pylori resistance to metronidazole, clarithomycin, amoxycillin, tetracycline and trovafloxacin in the Netherlands. $J$ Antimicrob Chemother 1999; 43: 511-515.

4. Mégraud F, Lehn N, Lind T et al. Antimicrobial susceptibility testing of Helicobacter pylori in a large multicenter trial: the MACH 2 study. Antimicrob Agents Chemother 1999; 43: 2747-2752.

5. Houben MHMG, Van Der Beek D, Hensen EF, Craen AJM, Rauws EAJ, Tytgat GNJ. A systematic review of Helicobacter pylori eradication therapy - the impact of antimicrobial resistance on eradication rates. Aliment Pharmacol Ther 1999; 13: 1047-1055.

6. Piccolomini R, di Bonaventura G, Catamo G, Carbone F, Neri M. Comparative evaluation of the $\mathrm{E}$ test, agar dilution, and broth microdilution for testing susceptibilities of Helicobacter pylori strains to 20 antimicrobial agents. J Clin Microbiol 1997; 35: $1842-1846$.

7. Occhialini A, Urdaci M, Doucet-Populaire F, Bébéar CM, Lamouliatte H, Megraud F. Macrolide resistance in Helicobacter pylori: rapid detection of point mutations and assays of macrolide binding to ribosomes. Antimicrob Agents Chemother 1997; 41: 2724-2728.

8. Peitz U, Hackelsberger A, Malfertheiner P. A practical approach to patients with refractory Helicobacter pylori infection, or who are re-infected after standard therapy. Drugs 1999; 57: 905-920.

9. Boyanova L, Spassova Z, Krastev Z et al. Characteristics and trends in macrolide resistance among Helicobacter pylori strains isolated in Bulgaria over four years. Diagn Microbiol Infect Dis 1999; 34: 309-313.

10. Teare L, Peters T, Saverymuttu S, Owen R, Tiwari I. Antibiotic resistance in Helicobacter pylori. Lancet 1999; 353: 242.

11. Goodwin A, Kersulyte D, Sisson G, Veldhuyzen van Zanten SJ, Berg DE, Hoffman PS. Metronidazole resistance in Helicobacter pylori is due to null mutations in a gene $(r d x A)$ that encodes an oxygen-insensitive NADPH nitroreductase. $\mathrm{Mol}$ Microbiol 1998; 28: 383-393.

12. Wolle K, Nilius M, Leodolter A, Müller WA, Malfertheiner P, König W. Prevalence of Helicobacter pylori resistance to several antimicrobial agents in a region of Germany. Eur $J$ Clin Microbiol Infect Dis 1998; 17: 519-521.

13. Lopez-Brea M, Domingo D, Sanchez I, Alarcon T. Evolution of resistance to metronidazole and clarithromycin in Helicobacter pylori clinical isolates from Spain. J Antimicrob Chemother 1997; 40: 279-281.

14. Boyanova L, Stancheva I, Spassova Z, Katzarov N, Mitov I, Koumanova R. Primary and combined resistance to four antimicrobial agents in Helicobacter pylori in Sofia, Bulgaria. J Med Microbiol 2000; 49: 415-418.

15. Osato MS, Reddy R, Graham DY. Metronidazole and clarithromycin resistance amongst Helicobacter pylori isolates from a large metropolitan hospital in the United States. Int $J$ Antimicrob Agents 1999; 12: 341-347.

16. Glupczynski Y, Goutier S, Van den Borre C, Butzler J-P, Burette A. Surveillance of Helicobacter pylori resistance to antimicrobial agents in Belgium from 1989 to 1994. Gut 1995; 37 (suppl 1): A56.

17. Versalovic J, Shortridge D, Kibler K et al. Mutations in $23 \mathrm{~S}$ rRNA are associated with clarithromycin resistance in Helicobacter pylori. Antimicrob Agents Chemother 1996; 40: 477-480.

18. Franzin L, Pennazio M, Cabodi D, Rossini FP, Gioannini P. Clarithromycin and amoxicillin susceptibility of Helicobacter 
pylori strains isolated from adult patients with gastric or duodenal ulcer in Italy. Curr Microbiol 2000; 40: 96-100.

19. Kim JJ, Reddy R, Lee $\mathrm{M}$ et al. Analysis of metronidazole, clarithromycin and tetracycline resistance of Helicobacter pylori isolates from Korea. J Antimicrob Chemother 2001; 47: 459-461.

20. van Doorn L-J, Debets-Ossenkopp YJ, Marais A et al. Rapid detection by PCR and reverse hybridization of mutations in the Helicobacter pylori $23 \mathrm{~S}$ rRNA gene, associated with macrolide resistance. Antimicrob Agents Chemother 1999; 43: 1779-1782.

21. Dore MP, Osato MS, Realdi G, Mura I, Graham DY, Sepulveda AR. Amoxicillin tolerance in Helicobacter pylori. J Antimicrob Chemother 1999; 43: 47-54. 